\title{
Binary Sequences for Multiple Access Collision Channel: Identification and Synchronization
}

\author{
Yijin Zhang, Kenneth W. Shum, Member, IEEE, Wing Shing Wong, Fellow, IEEE, and Feng Shu, Member, IEEE
}

\begin{abstract}
In this paper we investigate the identification and synchronization problems on a multiple access collision channel. Following Massey's lead, solutions to these problems are addressed by protocol sequences. This paper considers two different levels of user synchroneity: frame-synchronous access and slotsynchronous access. For the identification problem, we study user-detectable sequences. These are sequences with the crosscorrelation property that allows each active user be detected within a bounded delay basing only on the channel activity information observed. Furthermore, we investigate the synchronization problem for delay-detectable sequences under the slotsynchronous access assumption. The goal of the synchronization problem is to determine the offset relations among all the active users. Sequences that allow such determination can be viewed as a special subset of user-detectable sequences. For both of these sequence families, it is desirable that the sequence length should be as short as possible. Hence, it is important to derive the minimum sequence lengths for these respective families. This is an extremely difficult open problem. Nevertheless, lower and upper bounds on these minimum lengths are presented in this paper under different levels of synchroneity assumptions. In addition, the performance of these sequences is demonstrated via numerical simulation.
\end{abstract}

Index Terms-Collision channel without feedback, protocol sequences, superimposed code, user-irrepressible sequences, optical orthogonal code.

\section{INTRODUCTION}

\section{A. Motivation}

$\mathbf{M}$ ASSEY and Mathys introduced a model of the multiple access collision channel without feedback in [1] and [2]. The model involves $T$ potential users aiming to transmit to a single receiver over a time-slotted channel. It is assumed that at most $M(M \leq T)$ users may be active at

Manuscript received May 7, 2013; revised October 11 and November 28, 2013. The editor coordinating the review of this paper and approving it for publication was C. da Silva.

This work was partially supported by the National Natural Science Foundation of China (No. 61301107, 61174060 and 61271230), the Specialized Research Fund for the Doctoral Program of Higher Education of China (No. 20133219120010), the Direct Grant of the Chinese University of Hong Kong (No. 4055013), and the Open Research Fund of National Mobile Communications Research Laboratory, Southeast University (No. 2013D02). This paper was presented in part at ISWCS'10, York, September 19-22, 2010.

Y. Zhang and F. Shu are with the School of Electronic and Optical Engineering, Nanjing University of Science and Technology, Nanjing, China. F. Shu is also with the National Mobile Communications Research Laboratory, Southeast University, China (e-mail: yijin.zhang@gmail.com; shufeng@njust.edu.cn).

K. W. Shum is with the Institute of Network Coding, The Chinese University of Hong Kong, Shatin, N. T., Hong Kong (e-mail: wkshum@inc.cuhk.edu.hk).

W. S. Wong is with the Department of Information Engineering, The Chinese University of Hong Kong, Shatin, N. T., Hong Kong (e-mail: wswong@ie.cuhk.edu.hk).

Digital Object Identifier 10.1109/TCOMM.2013.121313.130331 the same time. The signal transmitted by each user is modeled by a fixed-length packet, which occupies exactly one time slot. If two or more users send simultaneously, a collision occurs and all involved packets are lost. If exactly one user transmits, the packet can be received correctly.

In multiple access transmission, three tasks are commonly encountered [3]-[6]:

(i) to detect active users (identification),

(ii) to determine the sender and value of each successful received packet (decoding), and

(iii) to find their delay offsets (synchronization).

In this paper we investigate the identification problem and the identification-cum-synchronization problem on a collision channel. The latter is also known as signature coding. Since this paper does not deal with the coding/decoding issues, we will assume that there is no user payload in a packet. This is not simply a matter of convenience. In fact, in the next subsection we present applications examples which require no user payload and can be modeled as a signature coding problem. In such a model, at each time slot each user either transmits a signal pulse or else remains silent. So the length of a packet in a time slot can be regarded as 1 bit and we can define the channel-activity signal of the collision channel, $c(t)$, as follows:

$$
c(t)= \begin{cases}0 & \text { if no user transmits at slot } t \\ 1 & \text { if exactly one user transmits at slot } t \\ * & \text { if more than one user transmit at slot } t .\end{cases}
$$

As presented in [3]-[5], the identification and synchronization problems are addressed by assigning each user a distinct deterministic binary sequence rather than random coding. We call it a protocol sequence [2]. An active user transmits if the value " 1 " is read in the assigned sequence and keeps silent if " 0 " is read. It is possible for an active user to become inactive and vice versa. However, we assume that an inactive user must remain inactive for at least the length of a protocol sequence before becoming active. It is noted that such solution also applies in the OR channel paper [7] as well as for the signature coding papers [6], [8], [9]. In this paper, we use the word "length" and "period" of a sequence interchangeably.

Furthermore, the following two different levels of synchroneity are studied:

(i) Frame-synchronous access: each active user starts its sequence period at the same slot;

(ii) Slot-synchronous access: each active user starts its sequence period at the beginning of a time slot that is chosen randomly so that the offset delay between any two overlapping active users is uniformly distributed. 
To the authors' knowledge, in the literature there is no study on the minimum length of sequences which can be used to address the identification and synchronization problems of a collision channel. This paper is an attempt to investigate the lower and upper bounds of the minimum length under framesynchronous or slot-synchronous access assumption. Effects of the assumption on synchroneity are also examined.

\section{B. Applications}

The identification problem plays a fundamental role in many practical scenarios, some of which relating to wired communication were mentioned in [8], [9]. More examples can be found in [9] including file retrieval, login in mobile telecommunications systems and non-adaptive hypergeometric group testing.

Sensor alarm. Consider an area monitored by a sensor network which consists of several alarm sensors and one data sink. If one or more intruders enter the area, multiple alarms may be triggered. Solving the identification problem arising from the sensor signature coding problem enables the data sink to determine more precisely the locations where the intrusions have occurred.

Another interesting potential application of the identification problem is multilevel expurgated pulse-position modulation (MEPPM) [10] for visible light communications (VLC).

MEPPM. In MEPPM for VLC, the low-cost LED is exploited. Balanced incomplete block designs (BIBD) are used to construct the symbol alphabet of an LED array. It is assumed that all LEDs are frame-synchronous. The receiver can distinguish multiple power levels of light intensity and its task is to demodulate the received signal to the original BIBD codewords. In a collision channel the receiver can detect three levels of energy: $\{0,1, *\}$. Thus the identification problem in a frame-synchronous system discussed in this paper can be employed to address the MEPPM decoding.

For the synchronization problem, one application is target tracking by detecting energy pulses coming from multiple distributed sources. This application was introduced in [5].

Tracking. In a multi-static radar system, each transmitting source sends multiple pulses according to its assigned protocol sequence. If the sequences have the synchronization ability, then the detector can capture the exact sending time of each transmitting source's pulse train. The information can be utilized for more accurate tracking of the targets.

\section{Related Work}

For a collision channel, there is no study focusing merely on the identification and synchronization problems. They are usually investigated together with packet decoding. (See [3][5], [11], [12].) L. Györfi and S. Györi presented asymptotic lower and upper bounds on the minimum sequence length for frame-synchronous access in [11]. They further gave the bounds for slot-synchronous access in [12]. However, the proof relies on the random coding rather than deterministic protocol sequences and thus the results only hold for $T$ approaching infinity. References [3]-[5] provided several concrete constructions for slot-synchronous access, but decoding and throughput are the primary objects of investigation.
The notion of identification under frame-synchronous access is also addressed in another context for an OR channel, under the name superimposed code [8] and cover-free families [13]. There are only two values, 0 and 1 , in the channel-activity signal of the OR channel considered signifying whether a time slot contains no entry of value " 1 " or at least one such entry. Interested readers are referred to [9] for a survey of known results in the literature. The same problem has also been extensively studied in the adder channel [14], [15] in which a tight asymptotic lower bound $2 M / \log M$ on the minimum sequence length was established. The channel-activity signal of an adder channel includes different $M+1$ integers signifying exactly the number of transmitted users in a time slot. From the channel-activity signal, one sees both of these problems are very different from the identification problem under frame-synchronous access for a collision channel as discussed here. For slot-synchronous access, an asymptotic upper bound for $T$ approaching infinity on minimum sequence length was derived in [6] for both the synchronization and identification problems in an OR channel.

\section{Key Contributions}

The special case $T=M \geq 2$ is considered in this paper, as adopted in [2]. For the identification problem, our goal is to find protocol sequences that allow any user be detected from the channel-activity signal if and only if it has become active. Such a protocol sequence set is said to be user-detectable (UD). For the synchronization problem, a sequence set is said to be delay-detectable (DD) if it allows the relative delay offset of any active user be detected from the channel-activity signal. We note that the synchronization problem is trivially solved under the frame-synchronous access assumption, since all active users start their sequences at the same slot. In other words, a UD sequence set must be DD in this case.

The following are three major objects of this paper:

(i) $L_{\text {syn }}(M)$, the smallest sequence length of a UD sequence set for $M$ users under the frame-synchronous assumption;

(ii) $L_{\text {asyn }}(M)$, the smallest length of a UD sequence set for $M$ users under the slot-synchronous assumption;

(iii) and $L_{\text {asyn }}^{d}(M)$, the smallest length of a DD sequence set for $M$ users under the slot-synchronous assumption.

We also summarize the key contributions as follows:

(1) In Section III, we establish a lower bound on $L_{\text {syn }}(M)$. An upper bound and its corresponding construction are also given.

(2) Lower and upper bounds on $L_{a s y n}(M)$ are presented in Section IV. In addition, we provide a detecting algorithm by which the receiver can detect each active user.

(3) In Section V, we establish an upper bound on $L_{\text {asyn }}^{d}(M)$. We also propose an algorithm for solving the synchronization problem.

(4) Simulation results are presented in Section VI in order to provide information on the performance of the proposed identification algorithm.

\section{Definitions And Notations}

Given a binary sequence $s(t), t=0,1, \ldots, L-1$, of length $L$, we define its Hamming weight as $w(s):=\sum_{t=0}^{L-1} s(t)$. A 
TABLE I

KEY NOTATIONS AND DEFINITIONS.

\begin{tabular}{|c||c|}
\hline$w(s)$ & The Hamming weight of $s$ \\
\hline $\mathcal{I}_{s}$ & The characteristic set of $s$ \\
\hline$s^{(\tau)}$ & The cyclic shift of $s$ by $\tau$ \\
\hline$s_{1} \vee \ldots \vee s_{M}$ & The logical OR of $s_{1}, s_{2}, \ldots, s_{M}$ \\
\hline$s_{1} \preceq \bigvee_{i=2}^{M} s_{i}^{\left(\tau_{i}\right)}$ & $s_{1}$ is blocked by $s_{2}, \ldots, s_{M}$ \\
\hline$s_{1} \uplus \ldots \uplus s_{M}$ & The erasure sum of $s_{1}, s_{2}, \ldots, s_{M}$ \\
\hline$H_{s_{1} s_{2}}$ & The Hamming crosscorrelation between $s_{1}$ and $s_{2}$ \\
\hline$H_{s_{1}} s_{1}$ & The Hamming autocorrelation of $s_{1}$ \\
\hline
\end{tabular}

sequence set is said to be constant-weight if all sequences have the same Hamming weight.

Let the cyclic shift of a sequence $s$ by relative shift $\tau$ be denoted by $s^{(\tau)}(t):=s(t-\tau)$. The subtraction $t-\tau$ is performed modulo $L$.

A sequence can also be represented by a characteristic set $\mathcal{I}$, which is defined as the set of all time indices in a period where the value of the protocol sequence is equal to 1 . Cyclic shift of a sequence by integer $\tau$ is equivalent to adding $\tau$ modulo $L$ to the corresponding characteristic set.

The logical $O R$ of $M$ binary sequences of length $L$ is defined as a binary sequence of length $L$ which has 0 in the $k$-th position where all sequences have 0 in this position, and 1 otherwise, for $k=0,1, \ldots, L-1$. We denote the logical OR of $s_{1}, s_{2}, \ldots, s_{M}$ by $s_{1} \vee s_{2} \vee \ldots \vee s_{M}$.

We write $s_{1} \preceq\left(s_{2} \vee \ldots \vee s_{M}\right)$ if $s_{1} \vee s_{2} \vee \ldots \vee s_{M}=s_{2} \vee \ldots \vee$ $s_{M}$. The sequence $s_{1}$ is said to be blocked by $s_{2}, s_{3}, \ldots, s_{M}$, if we can find delay offsets $\tau_{i}$ for $i=2, \ldots, M$, such that $s_{1} \preceq \bigvee_{i=2}^{M} s_{i}^{\left(\tau_{i}\right)}$.

The erasure sum of $M$ binary sequences of length $L$ is defined as a sequence of length $L$ which has 0 in the $k$ th position where all sequences have 0 in this position, 1 if exactly one sequence has 1 in this position, and erasure symbol * otherwise, for $k=0,1, \ldots, L-1$. We denote the erasure sum of $s_{1}, s_{2}, \ldots, s_{M}$ by $s_{1} \uplus s_{2} \uplus \ldots \uplus s_{M}$.

Given two sequences $s_{1}(t)$ and $s_{2}(t)$, the Hamming crosscorrelation function between $s_{1}$ and $s_{2}$ is defined by

$$
H_{s_{1} s_{2}}(\tau):=\sum_{t=0}^{L-1} s_{1}(t) s_{2}(t-\tau)
$$

If $s_{1}(t)=s_{2}(t)$, the crosscorrelation is reduced to the autocorrelation of $s_{1}$ or $s_{2}$.

We list the key notations and definitions of this section in Table I. For illustration, consider the following sequence set:

$$
\begin{aligned}
& s_{1}=[10101010] \\
& s_{2}=[11001100] \\
& s_{3}=[10111000] .
\end{aligned}
$$

The characteristic set is $\mathcal{I}_{s_{1}}=\{0,2,4,6\}, \mathcal{I}_{s_{2}}=\{0,1,4,5\}$ and $\mathcal{I}_{s_{3}}=\{0,2,3,4\}$. We have $w\left(s_{1}\right)=w\left(s_{2}\right)=w\left(s_{3}\right)=4$. Also $s_{2}^{(2)}=[00110011]$ and $s_{1} \vee s_{2}^{(2)}=[10111011]$. Then we have $s_{3} \preceq s_{1} \vee s_{2}^{(2)}$ and thus $s_{3}$ is blocked by $s_{1}$ and $s_{2}$. One can also check that $s_{1} \uplus s_{2}=[* 110 * 110]$ and $H_{s_{1} s_{2}}(2)=2$.

\section{Frame-Synchronous ACCESS}

We make the following formal definition of a UD sequence set under frame-synchronous access.
Definition 1. A set of $M$ sequences is said to be UD under frame-synchronous access, if all erasure sums of up to $M$ sequences are distinct, i.e.,

$$
\biguplus_{i \in \mathcal{A}} s_{i} \neq \biguplus_{j \in \mathcal{B}} s_{j}
$$

for any subsets $\mathcal{A}, \mathcal{B} \subseteq\{1,2, \ldots, M\}$ and $\mathcal{A} \neq \mathcal{B}$.

By the above definition, a lower bound on sequence length can be computed simply by using the fact that since each erasure sum of at most $M$ sequences must be distinct, the total number of erasure sums in a UD set cannot exceed the number of $L$-digit ternary numbers. The total number of erasure sums we need to distinguish is $2^{M}$ because all subsets are possible, while the total number of observable channel outputs is $3^{L}$. Thus following $3^{L} \geq 2^{M}$ we have

$$
L_{\text {syn }}(M) \geq \frac{\log 2}{\log 3} M \approx 0.631 M .
$$

To prove a tighter lower bound on $L_{s y n}(M)$, we need the following definition and lemma.

Definition 2. Given a sequence set $\left\{s_{1}, s_{2}, \ldots, s_{M}\right\}$, if $s_{i}(k)=1$ and the erasure sum of $\left\{s_{1}, s_{2}, \ldots, s_{M}\right\} \backslash\left\{s_{i}\right\}$ at the $k$-th position is equal to 0 or 1 , then we say that the $k$-th position is exceptional for $s_{i}$. The collection of all exceptional positions of $s_{i}$ is denoted by $\mathcal{E}_{s_{i}}$. Obviously, we have $\mathcal{E}_{s_{i}} \subseteq \mathcal{I}_{s_{i}}$.

Lemma 1. If a sequence set $\mathcal{S}=\left\{s_{1}, s_{2}, \ldots, s_{M}\right\}$ is $U D$ under frame-synchronous access, then we have

(i) $\left|\mathcal{E}_{s_{i}}\right| \geq 1$ for any $i$;

(ii) $\mathcal{E}_{s_{i}} \neq \mathcal{E}_{s_{j}}$ for any $i \neq j$.

Proof: (i) Suppose $\left|\mathcal{E}_{s_{i}}\right|=0$, i.e., for all $t$ such that $s_{i}(t)=1$ the erasure sum of remaining sequences at time $t$ is $*$, then the following result holds:

$$
\biguplus_{u \in\{1,2, \ldots, M\} \backslash\{i\}} s_{u}=\biguplus_{u \in\{1,2, \ldots, M\}} s_{u} .
$$

This contradicts the definition of UD sequences for framesynchronous access in (1).

(ii) Suppose $\mathcal{E}_{s_{i}}=\mathcal{E}_{s_{j}}$ for some $i \neq j$. Let $e_{i}$ be the erasure sum

$$
e_{i}:=\biguplus_{u \in\{1,2, \ldots, M\} \backslash\{i\}} s_{u}
$$

and $e_{j}$ be the erasure sum

$$
e_{j}:=\biguplus_{u \in\{1,2, \ldots, M\} \backslash\{j\}} s_{u} .
$$

We will show that the sequence set $\mathcal{S}$ is not UD by proving $e_{i}=e_{j}$. Obviously, we have $e_{i}(t)=e_{j}(t)$ if $t$ is not in $\mathcal{I}_{s_{i}} \cup \mathcal{I}_{s_{j}}$. For $t \in \mathcal{I}_{s_{i}} \cup \mathcal{I}_{s_{j}}$, we have

$$
e_{i}(t)=e_{j}(t)= \begin{cases}1 & \text { if } t \in \mathcal{E}_{s_{i}}=\mathcal{E}_{s_{j}}, \\ * & \text { if } t \in \mathcal{I}_{s_{i}} \backslash \mathcal{E}_{s_{i}} \text { or } t \in \mathcal{I}_{s_{j}} \backslash \mathcal{E}_{s_{j}} .\end{cases}
$$

This completes the proof of Lemma 1. 
Example 1: The following is a UD sequence set under frame-synchronous access with $M=3, L=3$.

$$
\begin{aligned}
& s_{1}=[101] \\
& s_{2}=[111] \\
& s_{3}=[011]
\end{aligned}
$$

The first position is exceptional for both $s_{1}$ and $s_{2}$. The second is exceptional for both $s_{2}$ and $s_{3}$. The third is not exceptional for any sequence. One can check its structure is in accordance with Lemma 1.

We can establish the next theorem by means of Lemma 1 .

Theorem 2. For any $M \geq 2$,

$$
L_{\text {syn }}(M) \geq \frac{2 M}{3} \text {. }
$$

Proof: Denote $\left|\bigcup_{i \in\{1,2, \ldots, M\}} \mathcal{E}_{s_{i}}\right|$ by $n_{\mathcal{E}}$. Let $h$ be the number of the sequences which all have exactly one exceptional position. Then following (i) of Lemma 1 and the definition of exceptional position we have

$$
\sum_{i \in\{1,2, \ldots, M\}}\left|\mathcal{E}_{s_{i}}\right| \geq h+2(M-h) .
$$

On another hand, we also have

$$
\sum_{i \in\{1,2, \ldots, M\}}\left|\mathcal{E}_{s_{i}}\right| \leq 2 n_{\mathcal{E}}
$$

This inequality follows from the fact that the exceptional positions of all the sequences are located in $\bigcup_{i \in\{1,2, \ldots, M\}} \mathcal{E}_{s_{i}}$ and one exceptional position is occupied by at most two sequences simultaneously. After substituting (3) into (2), we further obtain

$$
h \geq 2 M-2 n_{\mathcal{E}} .
$$

Next we derive an upper bound on $h$. Suppose $h \geq n_{\mathcal{E}}+1$. By pigeonhole principle we always can find $\mathcal{E}_{s_{i}}=\mathcal{E}_{s_{j}}$ for some $i \neq j$. It contradicts (ii) of Lemma 1 and thus we have

$$
h \leq n_{\mathcal{E}} .
$$

After combining (4) and (5), we obtain $n_{\mathcal{E}} \geq 2 M / 3$. It implies $L \geq 2 M / 3$, since the length must be not smaller than $n_{\mathcal{E}}$.

For upper bound on $L_{\text {syn }}(M)$, we show a trivial construction for $M$ users with length $L=M$ : for $i=1,2, \ldots, M$, let $s_{i}(t)=1$ if and only if $t=i$. This sequence set enjoys the property (1), and thus we have

$$
L_{\text {syn }}(M) \leq M
$$

which is also the tight upper bound in OR channel [16]. To close the gap between $2 M / 3$ and $M$ for a collision channel is an interesting direction for future works.

\section{IDENTIFICATION FOR SLOT-SYNCHRONOUS ACCESS}

In subsequent discussion, we make use of the following formal definition of UD sequences under slot-synchronous access.

Definition 3. A set which has $M$ sequences of length $L$ is said to be UD under slot-synchronous access, if every erasure sum of up to $M$ sequences of any relative shifts is distinct from every other sum of $M$ or fewer sequences of any relative shifts, i.e.,

$$
\biguplus_{i \in \mathcal{A}} s_{i}^{\left(\tau_{i}\right)} \neq \biguplus_{j \in \mathcal{B}} s_{j}^{\left(\tau_{j}\right)}
$$

for any subsets $\mathcal{A}, \mathcal{B} \subseteq\{1,2, \ldots, M\}, \mathcal{A} \neq \mathcal{B},|\mathcal{A}| \geq|\mathcal{B}|$ and any integer $\tau_{1}, \tau_{2}, \ldots, \tau_{M}$. The defining property (1) under frame-synchronous access is a special case of (6) with $\tau_{1}=$ $\tau_{2}=\ldots=\tau_{M}=0$.

The objective of computing the minimum delay is complicated. In order to provide a deeper understanding of it, we present in this section lower and upper bounds on $L_{a s y n}(M)$.

\section{A. Lower Bound on Minimum period}

We obtain $L_{\text {asyn }}(2) \geq 2$ from Theorem 2 since framesynchronous access is a special case of slot-synchronous access. The lower bound in this case can be achieved through assigning $s_{1}=[11], s_{2}=[10]$. Furthermore, we will use the following result from [17] to derive a lower bound on $L_{\text {asym }}(M)$ for $M>2$.

Theorem 3. [17] Let $\mathcal{S}^{\prime}$ be a set of $M^{\prime}$ (not necessarily constant-weight) binary sequences of length $L$. If $L<$ $8\left(M^{\prime}\right)^{2} / 9$, then there exist the relative delay offsets of the sequences such that the sequence with minimum Hamming weight in $\mathcal{S}^{\prime}$ is blocked by the other sequences in $\mathcal{S}^{\prime}$.

From Theorem 3, we can deduce

Theorem 4. For any $M \geq 3$, we have

$$
L_{\text {asyn }}(M) \geq \frac{8\lceil M / 2\rceil^{2}}{9} .
$$

Proof: Let $\mathcal{S}$ be a sequence set consisting of sequences $s_{1}, s_{2}, \ldots, \ldots, s_{M}$, with sequence length strictly less than $8\lceil M / 2\rceil^{2} / 9$. After re-labeling the sequences if necessary, we assume without loss of generality that $s_{1}$ is a sequence of minimum Hamming weight in $\mathcal{S}$.

Let $M^{\prime}$ be the the integer $\lceil M / 2\rceil$, and consider the sequences $s_{1}, s_{2}, \ldots, s_{M^{\prime}}$. By Theorem 3 , we can arrange the relative delay offsets of these $M^{\prime}$ sequences such that $s_{1}$ is blocked by some shifted versions of $s_{2}, \ldots, s_{M^{\prime}}$, i.e., there exist $\tau_{2}, \tau_{3}, \ldots, \tau_{M^{\prime}}$ such that

$$
s_{1} \preceq s_{2}^{\left(\tau_{2}\right)} \vee s_{3}^{\left(\tau_{3}\right)} \vee \cdots \vee s_{M^{\prime}}^{\left(\tau_{M^{\prime}}\right)} .
$$

Likewise, if we apply Theorem 3 to sequences

$$
s_{1}, s_{M^{\prime}+1}, s_{M^{\prime}+2}, \ldots, s_{M},
$$

we can find relative delay offsets $\tau_{M^{\prime}+1}, \tau_{M^{\prime}+2}, \ldots, \tau_{M}$ such that

$$
s_{1} \preceq s_{M^{\prime}+1}^{\left(\tau_{M^{\prime}+1}\right)} \vee s_{M^{\prime}+2}^{\left(\tau_{M^{\prime}+2}\right)} \vee \cdots \vee s_{M}^{\left(\tau_{M}\right)} .
$$

Hence, the erasure sum

$$
s_{1} \uplus s_{2}^{\left(\tau_{2}\right)} \uplus s_{3}^{\left(\tau_{3}\right)} \uplus \cdots \uplus s_{M}^{\left(\tau_{M}\right)}
$$

is the same as the erasure sum

$$
s_{2}^{\left(\tau_{2}\right)} \uplus s_{3}^{\left(\tau_{3}\right)} \uplus \cdots \uplus s_{M}^{\left(\tau_{M}\right)} .
$$


This violates the property (6) and thus proves that the sequence set $\mathcal{S}$ is not UD under slot-synchronous access.

Example 2: One can check the following sequence set with $M=3, L=4$ :

$$
\begin{aligned}
& s_{1}=[1100] \\
& s_{2}=[1010] \\
& s_{3}=[1110]
\end{aligned}
$$

is UD under slot-synchronous access, and its sequence length achieves the minimum value for $M=3$ in Theorem 4 .

\section{B. Upper Bound on Minimum period}

In this subsection, we will show some special classes of sequence sets that are UD under slot-synchronous access. We need to recall the following definition:

Definition 4. A sequence set is said to be user-irrepressible (UI) [18] if each sequence is not blocked by other sequences no matter what the relative offsets are.

Theorem 5. A sequence set is UD under slot-synchronous access if it is UI.

Proof: Suppose that a set of $M$ sequences is UI but not UD under slot-synchronous access. Then there exist two subsets $\mathcal{A}=\left\{s_{i_{1}}, s_{i_{2}}, \ldots, s_{i_{k}}\right\} \neq\left\{s_{j_{1}}, s_{j_{2}}, \ldots, s_{j_{l}}\right\}=\mathcal{B}$ such that

$$
s_{i_{1}}^{\left(\tau_{i_{1}}\right)} \uplus s_{i_{2}}^{\left(\tau_{i_{2}}\right)} \uplus \ldots \uplus s_{i_{k}}^{\left(\tau_{i_{k}}\right)}=s_{j_{1}}^{\left(\tau_{j_{1}}\right)} \uplus s_{j_{2}}^{\left(\tau_{j_{2}}\right)} \uplus \ldots \uplus s_{j_{l}}^{\left(\tau_{j_{l}}\right)}
$$

for some $\left\{\tau_{i_{1}}, \tau_{i_{2}}, \ldots, \tau_{i_{k}}\right\}$ and $\left\{\tau_{j_{1}}, \tau_{j_{2}}, \ldots, \tau_{j_{l}}\right\}$. It implies

$$
s_{i_{1}}^{\left(\tau_{i_{1}}\right)} \vee s_{i_{2}}^{\left(\tau_{i_{2}}\right)} \vee \ldots \vee s_{i_{k}}^{\left(\tau_{i_{k}}\right)} \preceq s_{j_{1}}^{\left(\tau_{j_{1}}\right)} \vee s_{j_{2}}^{\left(\tau_{j_{2}}\right)} \vee \ldots \vee s_{j_{l}}^{\left(\tau_{j_{l}}\right)} \text {. }
$$

Due to $\mathcal{A} \neq \mathcal{B}$ and $|\mathcal{A}| \geq|\mathcal{B}|$, we can always find a sequence, say $s_{u}, s_{u} \in \mathcal{A}$ but $s_{u} \notin \mathcal{B}$, such that

$$
s_{u}^{\left(\tau_{u}\right)} \preceq s_{j_{1}}^{\left(\tau_{j_{1}}\right)} \vee s_{j_{2}}^{\left(\tau_{j_{2}}\right)} \vee \ldots \vee s_{j_{l}}^{\left(\tau_{j_{l}}\right)} .
$$

This contradicts the definition of UI sequence set. Thus a UI sequence set must be UD under slot-synchronous access.

By means of Theorem 5, UD sequences under slotsynchronous access can be designed relying on the UI property. We introduce the shortest known general constructions below, which are both based on the Chinese remainder theorem (CRT). The mapping $f: \mathbb{Z}_{p q} \rightarrow \mathbb{Z}_{p} \oplus \mathbb{Z}_{q}$ defined by $f(a):=(a \bmod p, a \bmod q)$ is a bijection from $\mathbb{Z}_{p q}$ to $\mathbb{Z}_{p} \oplus \mathbb{Z}_{q}$ when $p$ and $q$ are relatively prime [19], and preserves addition and multiplication by integers.

CRT Construction [20]: Given $M$, we set $q$ to be $2 M-1$, and $p$ any prime larger than or equal to $M$ and relatively prime to $2 M-1$. For $j=0,1, \ldots, M-1$, we let $\mathcal{I}_{s_{j}}^{\prime}:=$ $\left\{(j y, y) \in \mathbb{Z}_{p} \oplus \mathbb{Z}_{2 M-1}: y=0,1, \ldots, M-1\right\}$ and obtain the characteristic sets $\mathcal{I}_{s_{j}}$, by taking the inverse image $f^{-1}\left(\mathcal{I}_{s_{j}}^{\prime}\right)$ for $j=0, \ldots, M-1$. These $M$ sequences compose a constantweight UI sequence set.

CRT $_{p}$ Construction [17]: This construction is a variation of CRT construction. We set $p$ any prime larger than or equal to $M$ and $q$ to be $2 p-2$. We let $\mathcal{I}_{s_{0}}^{\prime}:=\left\{(y, 0) \in \mathbb{Z}_{p} \oplus \mathbb{Z}_{2 p-2}\right.$ : $y=0,1, \ldots, p-1\}$ and $\mathcal{I}_{s_{j}}^{\prime}:=\left\{(j y, y) \in \mathbb{Z}_{p} \oplus \mathbb{Z}_{2 p-2}: y=\right.$ $0,1, \ldots, p\}$ for $j=1, \ldots, M-1$. These $M$ sequences form a UI sequence set, but not constant-weight.
TABLE II

THE SHORTEST KNOWN PERIOD OF UI SEQUENCES

\begin{tabular}{|c||c|}
\hline$M$ & Shortest known period \\
\hline \hline $2 \leq M \leq 6$ & $2^{M}$ \\
\hline$M=7$ & 84 \\
\hline$M=8$ & 153 \\
\hline$M \geq 9$, non-prime $M$ & $p_{M}(2 M-1)$ \\
\hline$M \geq 9, \operatorname{prime} M$ & $p_{M}\left(2 p_{M}-2\right)$ \\
\hline
\end{tabular}

The following upper bound is guaranteed by the CRT and $\mathrm{CRT}_{p}$ constructions.

Theorem 6. Let $p_{M}$ denote the smallest prime larger than or equal to $M$. For any $M \geq 3$, we have

$$
L_{a s y n}(M) \leq \min \left(p_{M}(2 M-1), p_{M}\left(2 p_{M}-2\right)\right) .
$$

For $2 \leq M \leq 6$, the shift-invariant property [21] produces the shortest known UI set of the period $2^{M}$. For $M=8$, the shortest known period is obtained by computer searching. However, in the other cases, CRT and $\mathrm{CRT}_{p}$ constructions yield the shortest known sequence length for non-prime $M$ and prime $M$, respectively. We list them in Table II.

We have proved in [17] that the period of UI sequence set with $M$ sequences is at least $8 M^{2} / 9$. Thus UD sequence sets under slot-synchronous access which are not UI with sequence length between $8\lceil M / 2\rceil^{2} / 9$ and $8 M^{2} / 9$ may be a potential direction for seeking sequence sets with shorter period. One can check the sequence set in Example 2 is UD under slotsynchronous access, but not UI.

\section{Detecting Algorithm}

Definition 5. We say that $c(t)$ is matched to $s_{i}$ at time $t_{0}$ if $\forall t=0,1, \ldots, L-1, s_{i}(t)=1 \Rightarrow c\left(t_{0}+t\right)=1$ or $*$. Let $\alpha_{i}$ be the smallest integer such that $s_{i}(t)=0$ if $\alpha_{i}<t \leq L-1$. The starting time $t_{i}^{s}$ is used to denote the time index when user $i$ becomes active, i.e., begins to send its sequence. The ending time $t_{i}^{s}+\alpha_{i}$ represents the time index of the last bit 1 in a sequence period of user $i$. The alarming time $t_{i}^{a}\left(t_{i}^{a} \geq t_{i}^{s}\right)$ is the time index when the first matching of user $i$ is detected by the receiver.

We now introduce the detecting algorithm used for a UI sequence set. The receiver observes the channel all the time and tracks the set of active users by $M$ Boolean variables active $_{i}$ for $i=1,2, \ldots, M$. The values are set to FALSE initially. In each slot, the receiver checks whether $c(t)$ is matched to $s_{i}$ or not. To determine whether $c(t)$ is matched to $s_{i}$ at some time index $t_{0}$, it is necessary for the receiver to know all values of $c\left(t_{0}\right), c\left(t_{0}+1\right), \ldots, c\left(t_{0}+\alpha_{i}\right)$. The receiver would make the decision at $t_{0}+\alpha_{i}$. If there is a matching at $t_{0}$, then active $_{i}$ is set to TRUE. Otherwise, we would set active $e_{i}$ as FALSE. In other words, whether there is a matching with $s_{i}(t)$ is determined slot by slot through a sliding window of $\alpha_{i}+1$ slots. We summarize the procedure in Algorithm 1.

Theorem 7. Assign a UI sequence set of $M$ sequences to user 1 to $M$. Then under slot-synchronous access Algorithm 1 can 


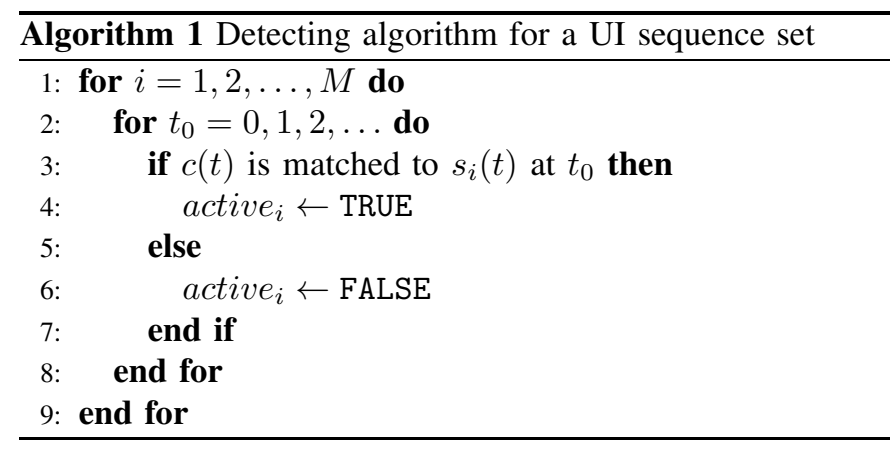

successfully identify user $i$ not later than $t_{i}^{s}+\alpha_{i}$ if and only if user $i$ becomes active at $t_{i}^{s}$.

Proof: Consider user $i$ and its assigned sequence $s_{i}$. Let it become active at $t_{i}^{s}$. Suppose there exists a matching of $s_{i}$ at $t_{0}$ with $t_{0}>t_{i}^{s}+\alpha_{i}$ or $t_{0}<t_{i}^{s}-\alpha_{i}$. By the condition of $t_{0}$, we know user $i$ actually does not transmit in the time interval $\left[t_{0}, t_{0}+\alpha_{i}\right]$. Hence we find all non-zeros in $\left[t_{0}, t_{0}+\alpha_{i}\right]$ are contributed by some other users. By the definition of matching, we further obtain that $s_{i}$ can be blocked by other sequences, which contradicts the condition that the assigned sequence set is UI. Thus the matching of $s_{i}$ can only possibly exist in $\left[t_{i}^{s}-\alpha_{i}, t_{i}^{s}+\alpha_{i}\right]$. This suffices to show that user $i$ would be identified by Algorithm 1 only if it has become active. On another hand, the receiver can always find $c(t)$ is matched to $s_{i}(t)$ at $t_{i}^{s}$, i.e., identify user $i$ at $t_{i}^{s}+\alpha_{i}$. Therefore we can conclude that Algorithm 1 can successfully identify the active user $i$ with $\alpha_{i}$ slots delay in the worst case.

Example 3: By the CRT construction, for $M=3$ we can design the UD sequence set under slot-synchronous access with $L=15$ below:

$$
\begin{aligned}
& s_{1}=[100000100000100] \\
& s_{2}=[111000000000000] \\
& s_{3}=[100010001000000] .
\end{aligned}
$$

If the starting time of these users are 0,6 and 5, respectively, we have the following case to make the user-detection. Sequence periods are indicated by underbrace.

User 1: $\underbrace{10000010000010010000010000010000000}$

User 2: $0000001 \underbrace{11100000000000000000000000000}$

User 3: $000001 \underbrace{100010001000000100010001000000}$

$$
c(t): 10000 \underline{1 * \overline{1} 11} 0011010000110010011000000
$$

One can check $c(t)$ is matched to $s_{2}(t)$ at $t=5,6,7$, respectively, which are indicated by underline. Since $\alpha_{2}=2$, the receiver would set active $_{2}$ as TRUE when $t=7,8,9$, which are indicated by overline. Thus the receiver cannot know the exact starting time of user 2 . The detecting procedure is presented in Fig. 1.

\section{SynCHRonizATION FOR SLOT-SynCHRONOUS ACCESS}

We present the formal definition of DD sequence set under slot-synchronous access as follows.

Definition 6. A set which has $M$ sequences of length $L$ is said to be DD under slot-synchronous access, if every erasure

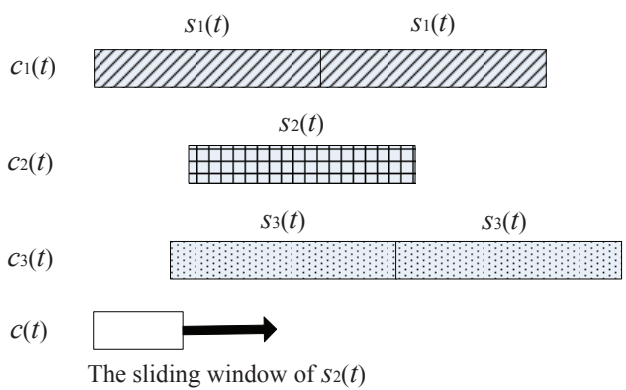

Fig. 1. The receiver tries to match $c(t)$ with $s_{2}$.

sum of up to $M$ sequences of some relative shift is distinct from (i) the same sequence set with any other relative shift and (ii) every other sum of $M$ or fewer sequences of any relative shifts, respectively, i.e.,

$$
\biguplus_{i \in \mathcal{A}} s_{i}^{\left(\tau_{i}\right)} \neq \biguplus_{j \in \mathcal{B}} s_{j}^{\left(\tau_{j}^{\prime}\right)}
$$

for any subsets $\mathcal{A}, \mathcal{B} \subseteq\{1,2, \ldots, M\}$ with $|\mathcal{A}| \geq|\mathcal{B}|$ and any integer $\tau_{1}, \tau_{1}^{\prime}, \tau_{2}, \tau_{2}^{\prime}, \ldots, \tau_{M}, \tau_{M}^{\prime}$, such that $\mathcal{A} \neq \mathcal{B}$ or $\mathcal{A}=\mathcal{B}$ with $\left\{\tau_{i}: \forall i \in \mathcal{A}\right\} \neq\left\{\tau_{j}^{\prime}: \forall j \in \mathcal{B}\right\}$. Obviously, the UD property under slot-synchronous access is a special case here by setting $\mathcal{A} \neq \mathcal{B}$ in (7).

As a DD sequence set must be UD under slot-synchronous access, Theorem 4 gives us a lower bound on $L_{a s y n}^{d}(M)$ too. In this section we focus on its upper bound.

\section{A. Upper Bound on Minimum Period}

In order to obtain an upper bound on $L_{\text {asyn }}^{d}(M)$, a sufficient condition for the DD property is presented below.

Theorem 8. A sequence set is DD under slot-synchronous access if any sequence is not blocked by other sequences and its non-zero shifted version, i.e.,

$$
s_{i} \npreceq \bigvee_{j \in\{1,2, \ldots, M\}} s_{j}^{\left(\tau_{j}\right)}
$$

for any $i$ and any $\tau_{j}$ with $\tau_{i} \neq 0$.

Proof: We prove this theorem by contradiction. Suppose a sequence set satisfying (8) is not DD. Then for some $\mathcal{A} \neq \mathcal{B}$ or $\mathcal{A}=\mathcal{B}$ with $\left\{\tau_{i}: \forall i \in \mathcal{A}\right\} \neq\left\{\tau_{j}^{\prime}: \forall j \in \mathcal{B}\right\}$ we can find

$$
\biguplus_{i \in \mathcal{A}} s_{i}^{\left(\tau_{i}\right)}=\biguplus_{j \in \mathcal{B}} s_{j}^{\left(\tau_{j}^{\prime}\right)} .
$$

In the case of $\mathcal{A} \neq \mathcal{B}$, the equation (9) implies we can find one sequence, labeled as $s_{g}, g \in \mathcal{A}, g \notin \mathcal{B}$, such that $s_{g}^{\left(\tau_{g}\right)} \preceq \bigvee_{j \in \mathcal{B}} s_{j}^{\left(\tau_{j}^{\prime}\right)}$ since $s_{g}^{\left(\tau_{g}\right)} \preceq \bigvee_{i \in \mathcal{A}} s_{i}^{\left(\tau_{i}\right)} \preceq \bigvee_{j \in \mathcal{B}} s_{j}^{\left(\tau_{j}^{\prime}\right)}$. This further implies

$$
s_{g} \preceq \bigvee_{j \in \mathcal{B}} s_{j}^{\left(\tau_{j}^{\prime}-\tau_{g}\right)} .
$$

In the case of $\mathcal{A}=\mathcal{B}$, by the same reason we can also find a sequence, labeled as $s_{h}, h \in \mathcal{A}, \mathcal{B}$, such that

$$
s_{h} \preceq \bigvee_{j \in \mathcal{B}} s_{j}^{\left(\tau_{j}^{\prime}-\tau_{h}\right)}
$$


with $\tau_{h} \neq \tau_{h}^{\prime}$.

The above two results both violate (8). Thus we conclude that any sequence set satisfying (8) must be DD.

Definition 7. A family of binary sequences of length $L$ and Hamming weight $w$ that satisfies the following two properties:

(i) the value of Hamming autocorrelation function of any sequence is not bigger than $\lambda_{a}$ for any non-zero $\tau$ performed modulo $L$;

(ii) the value of Hamming crosscorrelation between any distinct two sequences is not bigger than $\lambda_{c}$ for any $\tau$,

is said to be an optical orthogonal code (OOC) $\left(L, w, \lambda_{a}, \lambda_{c}\right)[22]$.

Following Theorem 8, some known constructions of OOCs can be applied to design DD sequences if $w>(M-1) \lambda_{c}+\lambda_{a}$. We show here one example due to [23].

Theorem 9. [23] Let $p$ be any prime number and $m$ any positive integer. Then there exists an $\mathrm{OOC}\left(p^{2 m}-1, p^{m}+\right.$ $1,2,2)$ with $p^{m}-2$ codewords.

Example 4: For $M=4$, by setting $p=2$ and $m=3$ in Theorem 9, we obtain the following sequence set with $L=63$ :

$$
\begin{aligned}
& \mathcal{I}_{s_{1}}=\{0,4,7,17,27,30,34,39,58\} \\
& \mathcal{I}_{s_{2}}=\{0,5,8,14,15,34,53,54,60\} \\
& \mathcal{I}_{s_{3}}=\{0,8,21,22,24,26,27,40,48\} \\
& \mathcal{I}_{s_{4}}=\{0,5,10,16,28,30,43,45,57\}
\end{aligned}
$$

which can be found DD under slot-synchronous access since $w=2^{3}+1>3 \times 2+2$.

Next we single out a special case of Theorem 9 for general $M$ and give an upper bound on $L_{\text {asyn }}^{d}(M)$ which is roughly equal to $4 M^{2}$.

Theorem 10. Let $p_{2 M}$ denote the smallest prime larger than or equal to $2 M$. For any $M \geq 2$, we have

$$
L_{\text {asyn }}^{d}(M) \leq p_{2 M}^{2}-1 .
$$

Proof: By setting $m=1$ in Theorem 9, we know there exists an $O O C\left(p_{2 M}^{2}-1, p_{2 M}+1,2,2\right)$ with $M$ codewords as $p_{2 M}-2 \geq M$. Then we have $w=p_{2 M}+1>(M-1) \lambda_{c}+$ $\lambda_{a}$ by $\lambda_{a}=\lambda_{c}=2$. Following Theorem 8 , we thus obtain this OOC is DD under slot-synchronous access. The sequence length $p_{2 M}^{2}-1$ gives the upper bound in Theorem 10 .

\section{B. Detecting Algorithm}

The following algorithm was proposed in [3], [5], but indeed can be applied for any sequence sets satisfying Theorem 8 . The procedure is basically the same as that in Algorithm 1: sliding a window of $\alpha_{i}+1$ slots until there is a matching with $s_{i}$. Let the integral variable $s$ art $_{i}$ represent the detection result when user $i$ starts its new sequence period. If $c(t)$ is matched to $s_{i}$ at $t_{0}$, then we have start $_{i}=t_{0}$. We summarize the procedure in Algorithm 2 below.

Theorem 11. Assign a DD sequence set satisfying Theorem 8 to user 1 to M. Then under slot-synchronous access Algorithm

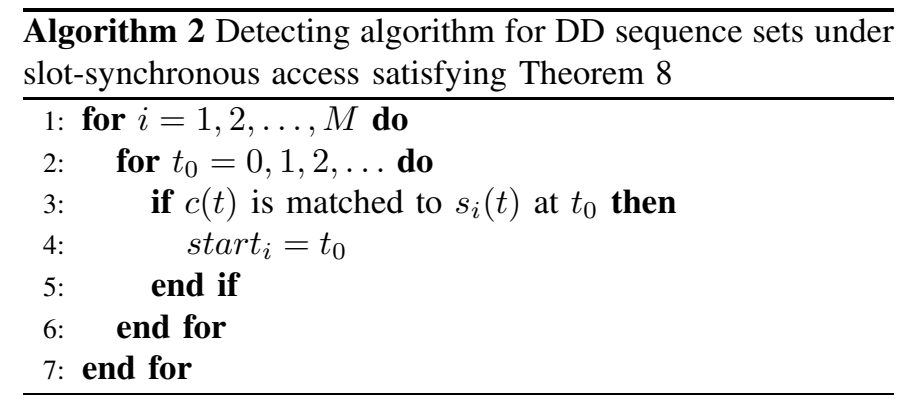

2 can determine the starting time of active user $i$ at $t_{i}^{s}+\alpha_{i}$ if it becomes active at $t_{i}^{s}$.

Proof: Consider that the starting time of active user $i$ is $t_{i}^{s}$, and is calculated as $t_{0}$ by Algorithm 2. This implies the matching of $s_{i}$ occurs at $t_{0}$. Suppose $t_{0} \neq t_{i}^{s}$. Then we know all non-zeros in $c\left(t_{0}\right), \ldots, c\left(t_{0}+\alpha_{i}\right)$ are contributed by other active sequences and a non-zero shifted version of $s_{i}$. Thus, we find $s_{i}$ can be blocked by other sequences and its non-zero shifted version, which contradicts Theorem 8 . We obtain that $t_{i}^{s}=t_{0}$, i.e., the exact starting time of user $i$ can be determined by Algorithm 2. Furthermore, by the definition of matching, the matching of $s_{i}$ at $t_{i}^{s}$ can only be detected at $t_{i}^{s}+\alpha_{i}$.

Example 4 continued: Let the four users in Example 4 begin to transmit their sequences, respectively, at $t=3,7,12,25$. Then following Theorem 11, we know the receiver at the time index $3+\alpha_{1}=61$ would detect the event that $c(t)$ is matched to $s_{1}$ at $t=3$, i.e., start $_{1}=3$ by Algorithm 2 .

\section{Performance Study}

We are interested in the latency which is measured as the time duration from the starting time of an active user to its alarming time. Obviously, the latency is upper bounded by the sequence period, which has been investigated extensively in Section III-V. Recall that $\alpha_{i}$ is the smallest integer such that $s_{i}(t)=0$ for all $t$ if $\alpha_{i}<t \leq L-1$. Under frame-synchronous access, it is easy to see that the receiver knows that user $i$ has become active exactly at $t_{i}^{s}+\alpha_{i}$. For synchronization under slot-synchronous access, by Theorem 11 we also obtain that the latency of user $i$ is exactly $\alpha_{i}$. However, for identification under slot-synchronous access, the alarming time of user $i$ may be earlier than $t_{i}^{s}+\alpha_{i}$ following Algorithm 1 and Theorem 7 . In order to explore the performance fluctuations of Algorithm 1 , we thus investigate the latency in this case by simulation.

In the simulation study, we assume that there are $M$ users and the CRT protocol sequences are used to specify their channel access permission time. Since users are not framesynchronized, there will be various combinations of starting time. In the simulation, we assume that the relative time shift between two users is uniformly distributed in their encountered minimum period $L=p_{M}(2 M-1)$.

Fig. 2 shows the starting time, alarming time and ending time of each user for $M=10$ in a simulation running. It is observed that for most users the alarming time is equal to the ending time, i.e., the latency is equal to $\alpha_{i}$. Here, we have $\left\{\alpha_{i}\right\}_{i=1}^{10}=\{198,9,193,197,191,199,195,196,192,180\}$ from the CRT construction. 


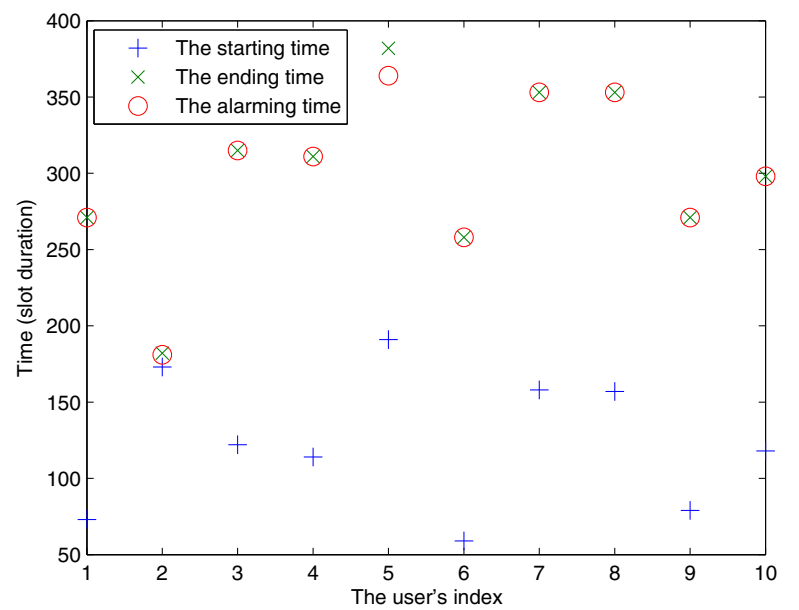

Fig. 2. The starting time, alarming time and ending time of each user in a simulation running for an identification problem with $M=10$ under slotsynchronous access.

TABLE III

THE AVERAGE PERFORMANCE OF IDENTIFICATION UNDER SLOT-SYNCHRONOUS ACCESS

\begin{tabular}{|c||c|c|c|c|}
\hline$M$ & $\rho$ & $\bar{\delta}$ & $\bar{\alpha}$ & $L$ \\
\hline \hline 9 & $5.6 \%$ & 150.5 & 151.4 & 187 \\
\hline 10 & $5.7 \%$ & 173.8 & 175.0 & 209 \\
\hline 11 & $5.6 \%$ & 195.6 & 196.8 & 231 \\
\hline 12 & $4.9 \%$ & 256.8 & 258.0 & 299 \\
\hline 13 & $5.1 \%$ & 282.6 & 283.8 & 325 \\
\hline 14 & $3.8 \%$ & 398.9 & 399.8 & 459 \\
\hline 15 & $3.9 \%$ & 435.1 & 436.3 & 493 \\
\hline 16 & $3.9 \%$ & 470.6 & 472.0 & 527 \\
\hline 17 & $3.9 \%$ & 504.5 & 505.9 & 561 \\
\hline 18 & $3.6 \%$ & 601.6 & 603.0 & 665 \\
\hline 19 & $3.6 \%$ & 639.5 & 640.9 & 703 \\
\hline 20 & $3.0 \%$ & 815.9 & 817.3 & 897 \\
\hline
\end{tabular}

Furthermore, we study the mean latency: $\frac{1}{M} \sum_{i=1}^{M}\left(t_{i}^{a}-t_{i}^{s}\right)$ of Algorithm 1 with respect to the user number. For each $M$ between 9 and 20, 100000 delay offset combinations are randomly generated. $\bar{\delta}$, the average mean latency over all these samples, is recorded in Table III compared with $\bar{\alpha}:=\frac{1}{M} \sum_{\underline{i=1}}^{M} \alpha_{i}$ and the sequence period $L$. Results obtained show that $\bar{\delta}<\bar{\alpha}<L$ for any $M$ and $\bar{\delta}, \bar{\alpha}, L$ all become larger when $M$ increases, as expected. We also compute the proportion $\rho$ for each $M$ : the ratio between the number of cases in which the latency of user $i$ is strictly smaller than $\alpha_{i}$ and that of all simulated cases: $100000 M$. Roughly speaking, it becomes smaller when $M$ increases as tabulated in Table III.

\section{CONCLUSION}

This paper has investigated UD and DD sequences to accordingly address the identification and synchronization problems on a collision channel. In order to study how deterministic guarantees can be offered, lower and upper bounds on their minimum periods are both presented for frame-synchronous and slot-synchronous access, respectively. We summarize these bounds for large $M$ in Table IV.

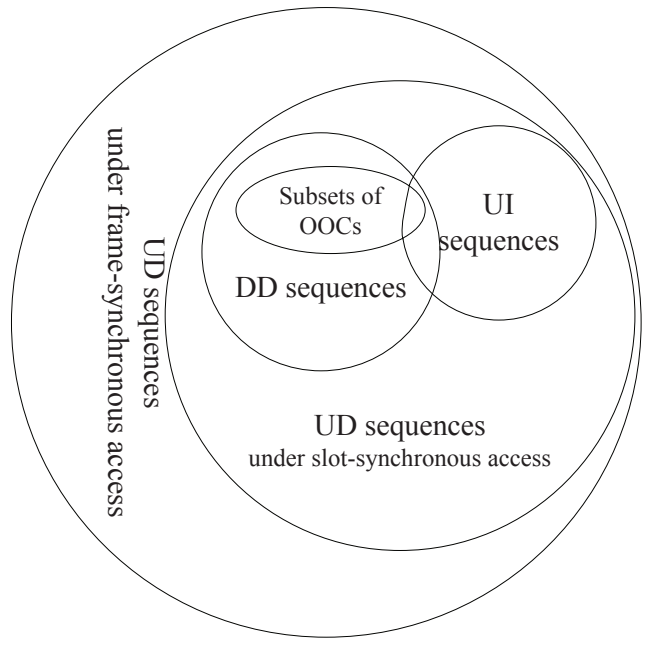

Fig. 3. Relationships among UD, DD sequences and other sequence designs.

TABLE IV

LOWER AND UPPER BOUNDS ON THE MINIMUM PERIOD OF UD AND DD SEQUENCES.

\begin{tabular}{|c||c|c|}
\hline & Lower bound & Upper bound \\
\hline \hline UD seq. under frame-syn. access & $2 M / 3$ (Thm. 2) & $M$ \\
\hline UD seq. under slot-syn. access & $2 M^{2} / 9$ (Thm. 4) & $2 M^{2}$ (Thm. 6) \\
\hline DD seq. under slot-syn. access & $2 M^{2} / 9$ (Thm. 4) & $4 M^{2}$ (Thm. 10) \\
\hline
\end{tabular}

As illustrated in Fig. 3, we establish some interconnections among UD, DD sequences and some other families of binary sequences. A UD sequence set under slot-synchronous access must be UD under frame-synchronous access by (1) and (6), and meanwhile a DD sequence set must be UD by (6) and (7). Furthermore, a UI sequence set is found UD from Theorem 6 and the OOCs with $w>(M-1) \lambda_{c}+\lambda_{a}$ can be used to construct DD sequences from Theorem 8 . These reported results open up many interesting directions for future research, for example, a construction of shorter UD sequences under slot-asynchronous access which is not UI.

In addition, we propose Algorithm 1 and Algorithm 2 that allow a receiver to solve the identification and synchronization problems within some bounded delay by employing UD and DD sequences, respectively. Simulation results are also provided to help readers come to a better understanding of their performance fluctuations.

\section{REFERENCES}

[1] J. L. Massey, "The capacity of the collision channel without feedback," in Proc. 1982 IEEE Int. Symp. Inform. Theory, p. 101.

[2] J. L. Massey and P. Mathys, "The collision channel without feedback," IEEE Trans. Inf. Theory, vol. 31, no. 2, pp. 192-204, Mar. 1985.

[3] N. Q. A, L. Györfi, and J. L. Massey, "Constructions of binary constantweight cyclic codes and cyclically permutable codes," IEEE Trans. Inf. Theory, vol. 38, no. 3, pp. 940-949, May 1992.

[4] L. Gyöfi and I. Vajda, "Construction of protocol sequences for multipleaccess collision channel without feedback," IEEE Trans. Inf. Theory, vol. 39, no. 5, pp. 1762-1765, Sep. 1993.

[5] K. W. Shum and W. S. Wong, "Construction and applications of CRT sequences," IEEE Trans. Inf. Theory, vol. 56, no. 11, pp. 5780-5795, Nov. 2010.

[6] S. Györi, "Signature coding for OR channel with asynchronous access," in Proc. 2005 IEEE Int. Symp. Inform. Theory, pp. 2040-2044. 
[7] A. R. Cohen, J. A. Heller, and A. J. Viterbi, "A new coding technique for asynchronous multiple access communication," IEEE Trans. Inf. Theory, vol. 19 , no. 5, pp. 849-855, Oct. 1971.

[8] W. H. Kautz and R. C. Singleton, "Nonrandom binary superimposed codes," IEEE Trans. Inf. Theory, vol. 10, pp. 363-367, Oct. 1964.

[9] S. Györi, "Coding for a multiple access OR channel: a survey," Discrete Applied Mathematics, no. 156, pp. 1407-1430, 2008.

[10] M. Noshad and M. Brandt-Pearce, "Multilevel pulse-position modulation based on balanced incomplete block designs," in Proc. 2012 IEEE Globecom, pp. 2930-2935.

[11] L. Györfi and S. Györi, "Bounds for multiple-access collision channel," in Proc. 2004 International Symposium on Information Theory and its Applications, pp. 1381-1386.

[12] — "Analysis of collision channel with asynchronous access," IEICE Trans. Fundamentals of Electronics, Commun. and Computer Sciences, vol. E88-A, no. 10, pp. 2878-2884, Oct. 2005.

[13] P. Erdős, P. Frankl, and Z. Fúredi, "Families of finite sets in which no set is covered by the union of $r$ others," Israel J. Mathematics, vol. 51, no. $1-2$, pp. 79-89, 1985.

[14] S. C. Chang and E. J. Weldon, "Coding for T-user multiple-access channels," IEEE Trans. Inf. Theory, vol. 25, no. 6, pp. 684-691, Nov. 1979.

[15] B. C. Lindström, "On a combinatory detection problem I," Publications of the Mathematical Institute of the Hungarian Academy of Science, vol. 9, pp. 195-207, 1964.

[16] A. Dyachkov and V. Rykov, "Bounds on the length of disjunctive codes," Problemy Peredachi Informatsii, vol. 18, no. 3, pp. 7-13, 1982.

[17] K. W. Shum, Y. Zhang, and W. S. Wong, "User-irrepressible sequences," in Proc. 2010 Conf. on Sequences and Their Applications, pp. 88-101.

[18] W. S. Wong, "New protocol sequences for random access channels without feedback," IEEE Trans. Inf. Theory, vol. 53, no. 6, pp. 20602071, Jun. 2007.

[19] K. Ireland and M. Rosen, A Classical Introduction to Modern Number Theory. Springer-Verlag, 1990

[20] K. W. Shum, W. S. Wong, C. W. Sung, and C. S. Chen, "Design and construction of protocol sequences: shift invariance and user irrepressibility," in Proc. 2009 IEEE Int. Symp. Inform. Theory, pp. 1368-1372.

[21] K. W. Shum, C. S. Chen, C. W. Sung, and W. S. Wong, "Shift-invariant protocol sequences for the collision channel without feedback," IEEE Trans. Inf. Theory, vol. 55, no. 7, pp. 3312-3322, Jul. 2009.

[22] F. R. K. Chung, J. A. Salehi, and V. K. Wei, "Optical orthogonal codes: design, analysis and applications," IEEE Trans. Inf. Theory, vol. 35, no. 3, pp. 595-604, May 1989 .

[23] H. Chung and P. V. Kumar, "Optical orthogonal codes-new bounds and an optimal construction," IEEE Trans. Inf. Theory, vol. 36, pp. 866-873, July 1990

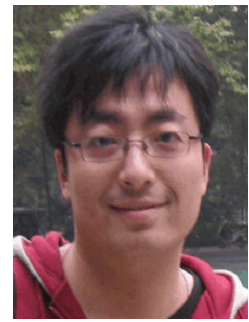

Yijin Zhang received the B.Eng. degree from Nanjing University of Posts and Telecommunications, China, in 2004, the M.S. degree from Southeast University, China, in 2007, and the Ph.D. degree in Information Engineering from the Chinese University of Hong Kong in 2010. He is now an associate professor in the School of Electronic and Optical Engineering, Nanjing University of Science and Technology. His research interests include coding theory and cooperative communication in wireless network.

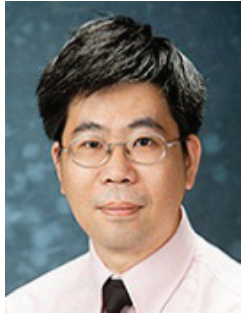

Kenneth W. Shum (M'00) received the B.Eng. degree in information engineering from the Chinese University of Hong Kong in 1993, and the M.S. and $\mathrm{Ph} . \mathrm{D}$. degrees in electrical engineering from the University of Southern California in 1995 and 2000, respectively. He is now a research associate professor with the Institute of Network Coding, the Chinese University of Hong Kong. He has been working on coding theory and its applications, including protocol sequences for multiple-access channels and network coding for distributed storage systems.

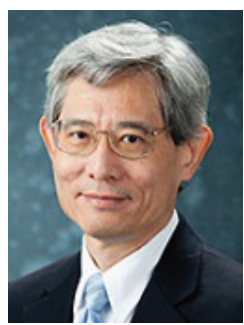

Wing Shing Wong (M'81-SM'90-F'02) received the combined master and bachelor degree (summa cum laude), from Yale University, New Haven, CT, in 1976, and the M.S. and Ph.D. degrees from Harvard University, Cambridge, MA, in 1978 and 1980, respectively.

After working at AT\&T Bell Laboratories, Holmdel, NJ, for ten years, in 1992, he has joined the Chinese University of Hong Kong, Shatin, and is now a Professor of Information Engineering. He was the Chairman of the Department of Information Engineering from 1995 to 2003 and is currently serving as the Dean of the Graduate School. He served as the Science Advisor at the Innovation and Technology Commission of the HKSAR Government from 2003 to 2005 . He is a Co-Editor-in-Chief of Communications in Information and Systems. $\mathrm{He}$ has participated in a variety of research areas including mobile communication systems, nonlinear filtering, search engine, and estimation and control of finite communication bandwidth systems.

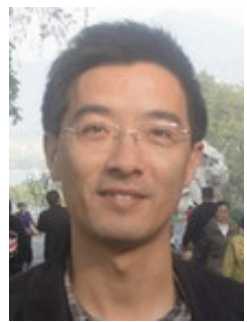

Feng Shu received his Ph.D. degree in signal and information processing from Southeast University, China, in 2002, M.S. degree in biological electronics engineering from Xidian University, China, in 1997, and B.S. degree in physics from Fuyang Normal College, China, in 1994. He is currently a full professor in the School of Electronic and Optical Engineering, Nanjing University of Science and Technology. His current research interest is in the areas of wireless communications, networking, and radar signal processing. 